\title{
Paramyotonia congenita of Von Eulenburg
}

INSERM

\section{Source}

INSERM. (1999). Orphanet: an online rare disease and orphan drug data base.

Paramyotonia congenita of Von Eulenburg. ORPHA:684

Paramyotonia congenita of Von Eulenburg is characterised by exercise- or cold-induced myotonia and muscle weakness. Prevalence is unknown. The syndrome is nonprogressive and is transmitted as an autosomal dominant trait. It is caused by mutations in the gene encoding the alpha subunit of the type IV voltage-gated sodium channel (SCN4A; 17q23.3). 